\title{
On the $T$-dependence of the magnetic penetration depth in unconventional superconductors at low temperatures: can it be linear?
}

\author{
N. Schopohl and O.V. Dolgov \\ Eberhardt-Karls Universität Tübingen \\ Institut für Theoretische Physik \\ Auf der Morgenstelle 14 \\ D-72076 Tübingen \\ Germany
}

\begin{abstract}
We present a thermodynamics argument against a strictly linear temperature dependence of the magnetic penetration depth, which applies to superconductors with arbitrary pairing symmetry at low temperatures.

74.25.Nf, 74.20Fg, 74.72.Bk.
\end{abstract}

Typeset using REVTEX 
Some evidence for an unconventional $d_{x^{2}-y^{2}}$-pairing symmetry in cuprate high $-T_{c}$ superconductors is provided by recent angle resolved photoemission experiments [1]. A striking proof for the $d_{x^{2}-y^{2}}$-symmetry of the Cooper pairs in cuprates arises from the observation of a spontanously generated half flux quantum in Josephson tunneling experiments carried out on tetracrystal substrates [2]. Early support for the possibility of a $d_{x^{2}-y^{2}}$-symmetry of the Cooper pairs in cuprate high $-T_{c}$ superconductors came from the observation of a linear $T$-dependence of the magnetic penetration depth [3], [4] at low temperatures $T$ :

$$
\lambda(T)-\lambda(0) \sim T
$$

command somewhere after the documentstyle command Such a linear $T$-dependence of the magnetic penetration depth (MPD) has a topological origin. If the order parameter associated with the Cooper pair condensate vanishes along node lines on the Fermi surface the spectrum $N_{s}(E)$ of quasiparticle excitations in the superconducting phase is gapless and varies proportional to $E$ at low excitation energies: $N_{s}(E) \sim E$ for $E \ll \Delta_{\max }$. For this reason a pure $d_{x^{2}-y^{2}}$-pairing state (node lines along $k_{x}= \pm k_{y}$ ) should display a strictly linear dependence of MPD vs. $T$ at low temperatures. In previous work this effect was also discussed for the polar phase in a triplet pairing superconductor, e.g. [5].

New experiments [6] indicate deviations from this linearity of MPD with temperature, for example a $T^{2}$-dependence of MPD below some cross over temperature $T^{*}$ was measured. Such a behaviour may occur due to various reasons. For example, I. Kosztin and A.J. Leggett [7] explaine this behavior in terms of nonlocal electrodynamics. Their argument is, that in clean $d_{x^{2}-y^{2}}$-pairing superconductors there exist surface induced non local effects, which lead to a $T^{2}$-dependence of $\lambda_{a b}(T)-\lambda_{a b}(0)$, as extracted from optical and microwave experiments with the magnetic field orientated parallel to the $\widehat{c}-$ direction. On the other hand, in experiments with the magnetic field orientated perpendicular to the $\widehat{c}$ - direction the $T$-dependence of MPD cannot be altered by the Kosztin-Leggett effect.

Since the Kosztin-Leggett effect [7] really depends on the existence of a surface in the problem it cannot be applied to other measurement techniques of MPD, for example direct static magnetic measurements, measurements of vortex properties, the lower critical magnetic field $B_{c 1}$, muon spin relaxation. Such techniques of measuring MPD have bulk character.

In the following we present a proof, for arbitrary superconductors, that a strictly linear $T$-dependence of MPD at low temperatures violates the third law of thermodynamics. For simplicity let us consider a uniform system where all properties depend on coordinates $\mathbf{r}-\mathbf{r}^{\prime}$ only. The current-current correlator,

$$
\eta(\mathbf{k}, \omega)=k^{2}-\frac{\omega^{2}}{c^{2}} \varepsilon_{t r}(\mathbf{k}, \omega)
$$

connects the vector potential $\mathbf{A}(\mathbf{k}, \omega)$ to the external current $\mathbf{j}_{\text {ext }}(\mathbf{k}, \omega)$ via

$$
\eta(\mathbf{k}, \omega) \mathbf{A}(\mathbf{k}, \omega)=\frac{4 \pi}{c} \mathbf{j}_{e x t}(\mathbf{k}, \omega)
$$

In turn, the transversal dielectric function, $\varepsilon_{t r}(\mathbf{k}, \omega)$, is related to the electromagnetic kernel $Q(\mathbf{k}, \omega)$ by the relation 


$$
\varepsilon_{t r}(\mathbf{k}, \omega)=1-\frac{4 \pi Q(\mathbf{k}, \omega)}{\omega^{2}}
$$

The definition of the operator of inverse MPD is then

$$
\frac{1}{\lambda^{2}(\mathbf{k}, T)}=\lim _{\omega \rightarrow 0} \frac{\omega^{2}}{c^{2}}\left\{1-\operatorname{Re} \varepsilon_{t r}(\mathbf{k}, \omega)\right\} \equiv \frac{4 \pi}{c^{2}} Q(\mathbf{k}, \omega=0)
$$

In the static case the additional free energy in the presence of an externaly controlled current distribution $\mathbf{j}_{\text {ext }}(\mathbf{k})$ (we use a transversal gauge) can be written in the form [8]:

$$
\begin{aligned}
\mathcal{F} & =-\frac{1}{2 c} \int \frac{d^{3} k}{(2 \pi)^{3}} \mathbf{j}_{\text {ext }}(\mathbf{k}) \cdot \mathbf{A}(-\mathbf{k}, \omega=0) \\
& =-\frac{1}{8 \pi} \int \frac{d^{3} k}{(2 \pi)^{3}} \eta(\mathbf{k}, \omega=0)|\mathbf{A}(\mathbf{k}, \omega=0)|^{2}
\end{aligned}
$$

By using these relations and Maxwell's equations it follows

$$
\mathcal{F}=-\frac{1}{8 \pi} \int \frac{d^{3} k}{(2 \pi)^{3}}\left[k^{2}+\frac{1}{\lambda^{2}(\mathbf{k}, T)}\right] \frac{|\mathbf{k} \times \mathbf{B}(\mathbf{k} ; T)|^{2}}{k^{4}}
$$

Here $\mathbf{B}(\mathbf{k}, T)$ is the (temperature dependent) induced magnetic field and satisfies the equation:

$$
\left[k^{2}+\frac{1}{\lambda^{2}(\mathbf{k}, T)}\right] \mathbf{B}(\mathbf{k} ; T)=\frac{4 \pi}{c} i \mathbf{k} \times \mathbf{j}_{\text {ext }}(\mathbf{k})
$$

Differentiating Eq.(7) with respect to temperature $T$ and calculating the derivative $\frac{\partial}{\partial T} \mathbf{B}(\mathbf{k} ; T)$ from Eq.(8) we get an expression for the entropy:

$$
S(T)=-\frac{\partial \mathcal{F}}{\partial T}=-\frac{1}{8 \pi} \int \frac{d^{3} k}{(2 \pi)^{3}} \frac{\partial}{\partial T}\left[\frac{1}{\lambda^{2}(\mathbf{k}, T)}\right] \frac{|\mathbf{B}(\mathbf{k} ; T)|^{2}}{k^{2}}
$$

According to the Nernst principle (third law of thermodynamics) the entropy should vanish in the limit $T \rightarrow 0$. From the positivity of the integrand we must conclude

$$
\lim _{T \rightarrow 0} \frac{\partial \lambda(\mathbf{k}, T)}{\partial T}=0
$$

If we wish to avoid a violation of the third law of thermodynamics the $T$-dependence of the magnetic penetration depth in a superconductor cannot be of the form $\lambda(T)-\lambda(0) \sim T^{n}$ with $n=1$. The argument can be extended to any nonuniform system.

We see that the vanishing of the first derivative of MPD for $T \rightarrow 0$ is a consequence of a general principle of thermodynamics. The value of $T^{*}$ below which a deviation of the linear $T$-dependence of MPD may be observed depends on the exact physical mechanism. It may be nonlocality [7], it may be the effect of impurities (as proposed in Ref. [9]), it may be also the effect of collective excitations (e.g. the influence of vertex corrections on the $T$-dependence of MPD was discussed in Ref. [10] for the case of pure $s$-wave pairing).

A famous reformulation of the third law of thermodynamics states that it is impossible to reach absolute zero. From this point of view a pure $d_{x^{2}-y^{2}}$-pairing symmetry in clean 
high- $T_{c}$ superconductors becomes, perhaps, invalid for $T \rightarrow 0$. A possibility to avoid the paradox of a linear $T$-dependence of MPD for $T \rightarrow 0$ in cuprate superconductors is a phase transition ( at a temperature $T_{c 2}$ much lower than the transition temperature $T_{c}$ ) to a new unconventional pairing state without nodes on the Fermi surface [11], [12].

Acknowledgements: It is a pleasure to thank A.J. Leggett for helpful correspondence and encouragement. Also we acknowledge useful discussions with R.P. Huebener, D. Rainer, K. Scharnberg, C.C. Tsuei and G.E. Volovik. 


\section{REFERENCES}

[1] H. Ding et al., Phys. Rev. B 54, 9678(1996).

[2] C.C. Tsuei et al., Nature 387,481(1997).

[3] W.N. Hardy et al., Phys. Rev. Lett. 70, 3999 (1993).

[4] D.A. Bonn et al., Phys. Rev. B 50, 4051 (1994).

[5] R.A. Klemm, K. Scharnberg, ,D.Walker and C.T. Rieck, Z.Phys. B72, 139 (1988).

[6] D.A. Bonn and W.N. Hardy, in Physical Properties of Hifh temperature Superconductors, ed. by D.M. Ginsberg ( World Scientific, Singapore, 1996), vol. 5.

[7] I. Kosztin and A.J. Leggett, Phys. Rev. Lett. 79, 135, (1997).

[8] O.V. Dolgov, D.A. Kirzhnits, V.V. Losyakov, Sov. Phys.-JETP, 56, 1095, (1982); D.A. Kirzhnits "General Properties of Electromagnetic Response Functions", Ch. 2 , Eq. (9.5) in: The Dielectric Function of Condensed Systems, eds., L.V. Keldysh, D.A. Kirzhnits, A.A. Maradudin, Elsevier Publ. (1989).

[9] J.P. Hirschfeld and N. Goldenfeld, Phys. Rev. B 48, 4219, (1993).

[10] G.M. Eliashberg, G.V. Klimovitch and A.V. Rylyakov, J. of Superconductivity 4, 393 (1991).

[11] R.B. Laughlin, Physica C 234, 280(1994).

[12] G. Preosti, H. Kim and P. Muzikar, Phys. Rev. B 50,13638(1994). 